\title{
Estudo comparativo de comunidades de briófitas sujeitas a diferentes graus de inundação no município de São Domingos do Capim, PA, Brasil
}

\author{
Moisés Oliveira Lopes ${ }^{1}$, Márcio Roberto Pietrobom ${ }^{1}$, Dimas Marchi do Carmo ${ }^{2}$ e \\ Denilson Fernandes Peralta2,3
}

Recebido: 15.07.2015; aceito: 18.02.2016

\begin{abstract}
Comparative study of communities of bryophytes in different degrees of flooding in the municipality of São Domingos do Capim, Pará State, Brazil). Third-five transects were analyzed $(10 \times 10 \mathrm{~m})$ for the floristic survey and eighteen transects (six for each vegetation type: Igapó Forest (IG), Várzea Forest (VA), and Terra Firme Forest (TE)) were randomly analyzed in order to compare the bryophyte communities. We found 118 species: 37 mosses and 82 liverworts Lejeuneaceae (63 species) was the richest among the liverwort families to liverworts and Calymperaceae (eight species) among mosses. The estimated species richness was lower than expected, but the collector's curve was stabilized. The most colonized substrate was live bark (101 spp., 45 exclusive) followed by decomposing bark (66 spp., 16 exclusive). The results show that the vegetation types analyzed have has differences in bryophyte community richness (IG $>$ TF $>$ VA) and composition, due to the occurrence of exclusive species. The cluster (UPGMA) and ordination (NMDS) analyses indicated the presence of community structure among the three phytophysiognomy.
\end{abstract}

Keywords: Amazon Forest, Bryophyta, Igapó Forest, Marchantiophyta

RESUMO - (Estudo comparativo de comunidades de briófitas sujeitas a diferentes graus de inundação no município de São Domingos do Capim, PA, Brasil). Foram analisadas 35 parcelas $(10 \times 10 \mathrm{~m})$ para a listagem florística e, destas, 18 parcelas (seis para cada fitofisionomia: Floresta de Igapó (IG), Floresta de Várzea (VA) e Floresta de Terra Firme (TE)) foram sorteadas para a análise de comparação das comunidades de briófitas. Encontramos 118 espécies de briófitas: 37 musgos e 82 hepáticas, sendo que Lejeuneaceae (63 espécies) foi a família mais rica para hepáticas e Calymperaceae (oito espécies) para musgos. A estimativa de riqueza está abaixo da esperada, porém a curva de coletor estabilizada. O substrato mais colonizado foi tronco vivo (101 spp., 45 exclusivas) seguido de tronco em decomposição (66 spp., 16 exclusivas). Os resultados obtidos mostram que as fitofisionomias analisadas apresentam diferenças na riqueza ( $\mathrm{IG}>\mathrm{TF}>\mathrm{VA})$ e composição das comunidades de briófitas, devido à existência de espécies exclusivas e as análises de Agrupamento (UPGMA) e Ordenação (NMDS) indicaram estruturação das comunidades entre as três fitofisionomias.

Palavras-chave: Amazônia, Bryophyta, Floresta de Igapó, Marchantiophyta

\section{Introdução}

No Estado do Pará ocorrem 329 espécies das 1.524 espécies de briófitas conhecidas para o Brasil (Costa \& Peralta 2015). Este Estado está completamente inserido no domínio fitogeográfico da Amazônia e possui extensa área territorial e diversidade de ambientes.

A Região Amazônica abriga a maior bacia hidrográfica do mundo, cobrindo cerca de seis milhões de $\mathrm{km}^{2}$ e 11.000 afluentes (MMA 2014). A água é vital para o desenvolvimento dos organismos e um dos principais recursos utilizados pelas briófitas, principalmente para a reprodução e, influencia diretamente na sua diversidade (Gradstein et al. 2001, Proctor 1982). A disponibilidade de água na Amazônia define as fitofisionomias circundantes, sendo a classificação da vegetação baseada em suas interações como: presença constante de alagamento (Igapó), alagamento sazonal (Várzea) e rápida drenagem (Terra Firme), entre outras.

Costa et al. (2011) discutem a diversidade dos musgos e Gradstein \& Costa (2003) das hepáticas no

1. Universidade Federal do Pará, campus Universitário de Bragança, Alameda Leandro Ribeiro s/n, Aldeia, 68600-000 Bragança, PA, Brasil

2. Instituto de Botânica, Av. Miguel Stéfano, 3687, 04301-012 São Paulo, SP, Brasil

3. Autor para correspondência: denilsonfperalta@gmail.com 
Bioma Amazônia, porém não apresentam informações sobre a diversidade e distribuição das espécies nas fitofisionomias que formam este Bioma e incluem, por exemplo: as Campinas, áreas de Floresta de Várzea, Terra Firme e Igapó.

Lisboa (1991) caracteriza as briófitas dentro do Projeto "Flora Amazônica" trazendo um histórico dos estudos neste Bioma, desde a primeira excursão em 1977 até 1983, e enfatiza que estes estudos são muito recentes e necessitam ser ampliados. Ainda assim, o Projeto "Flora Amazônica" permanece até o momento sem um diagnóstico da diversidade e endemismo das briófitas na Floresta Amazônica, esta análise é de extrema importância para valorizar e estimular os estudos na região. Análises deste nível já foram realizadas para vertebrados terrestres e, dessa maneira são conhecidas oito áreas de extrema importância para conservação e endemismo destes organismos na Amazônia (Silva et al. 2005).

No Estado do Pará o esforço de especialistas, e consequentemente o conhecimento das briófitas, está concentrado principalmente nas regiões próximas da capital e na Floresta Nacional de Caxiuanã (Santos 2006), onde ocorrem diferentes fitofisionomias Amazônicas: Floresta de Várzea, Floresta de Terra Firme, Floresta de Igapó, Capoeira, Vegetação Savanóide, Manguezal e Restinga (Lisboa \& Ilkiu-Borges 2001, Lisboa \& Ilkiu-Borges 2007, Alvarenga \& Lisboa 2009, Moraes \& Lisboa 2009). Esses trabalhos contribuíram significativamente com informações sobre a ocorrência e ecologia (tipos de hábitat e substratos colonizados) das comunidades de briófitas.

Realizando comparação entre ambientes de Terra Firme e "inundáveis" existem apenas os trabalhos de Alvarenga \& Lisboa (2009), que estudaram e compararam as Florestas de Terra Firme, Várzea, Capoeira, Igapó e Campina na Amazônia Oriental, encontrando diferença na distribuição das 120 espécies encontradas entre estas fitofisionomias, indicando a Floresta de Terra Firme como a mais rica (porém sem apresentar os valores encontrados para cada fitofisionomia analisada).

E, Benavides et al. (2004), que encontraram riqueza semelhante, porém grande diferença na composição das 109 espécies entre Floresta de Terra Firme (73 espécies) e "ambientes inundáveis" (79 espécies) na Amazônia Ocidental.

As briófitas submetidas à influência do alagamento sazonal na margem de grandes rios foram estudadas em diversos trabalhos ao redor do mundo (Glime 1968, Flowers 1973, Empain 1973, McFarland \& Wistendahl 1976), analisando aspectos da ecologia e sucessão, porém enfocando somente as briófitas terrícolas sendo impossível a comparação ou estabelecimento de padrões em grande escala.

Dentro deste cenário o objetivo deste trabalho foi comparar as comunidades de briófitas em três fitofisionomias com disponibilidade de água elevada e próximas geograficamente, distintas pela influência de alagamento: Floresta de Igapó (constante), Floresta de Várzea (sazonal) e Floresta de Terra Firme (ausência de alagamento).

\section{Material e métodos}

Área estudada - As áreas amostradas localizam-se no entorno das comunidades Coração de Jesus e Nova Aliança, localizadas entre a rodovia PA-127 e o Rio Capim, distantes 15 a $18 \mathrm{~km}$ do município de São Domingos do Capim, na região nordeste do Pará, e foram amostradas em novembro de 2012.

O clima é quente e úmido com temperatura média de $20{ }^{\circ} \mathrm{C}$, umidade relativa de $91 \%$ e a pluviosidade de $2.350 \mathrm{~mm}$ anuais, a vegetação é caracterizada principalmente por capoeiras em estado avançado de desmatamento, com árvores de menor porte e fragmentos de matas primárias bastante exploradas (IDESP 2015).

Nesta região foram amostradas três áreas próximas geograficamente e consideradas bem preservadas, para a implantação das parcelas e amostragem das briófitas, visando padronizar a luminosidade, temperatura, idade em termos sucessionais, altitude e disponibilidade de nutrientes. Estas áreas são representadas pelas fitofisionomias: Floresta de Igapó - constantemente alagada (IG), Floresta de Várzea alagada sazonalmente (VA) e Floresta de Terra Firme - não alagada (TE). A caracterização florística e física destas fitofisionomias segue Veloso et al. (1991).

Amostragem - As amostras foram coletadas com a metodologia usual para o grupo, seguindo Papp et al. (2011) até a estabilização da curva de coletor em cada fitofisionomia. Foram utilizadas 35 parcelas $(10 \times 10 \mathrm{~m})$ para a listagem florística e colonização de substratos (19 para IG, 6 para TE e 10 para VA) (tabela 1) e destas 18 parcelas (seis para cada fitofisionomia, pois havia seis para Terra Firme e as demais foram randomizadas) para a análise de similaridade florística 
A identificação das espécies seguiu a metodologia descrita por Costa et al. (2010) e foi baseada nos trabalhos de Sharp et al. (1994), Buck (1998), Gradstein \& Costa (2003) e Gradstein \& IlkiuBorges (2009). O sistema de classificação adotado foi Crandall-Stotler et al. (2009) para Marchantiophyta e Goffinet et al. (2009) para Bryophyta.

As amostras foram incorporadas nos herbários da Universidade Federal do Pará (UFPA), com duplicatas no Herbário do Estado "Maria Eneyda P. Kauffmann Fidalgo" (SP). As espécies foram classificadas de acordo com o substrato em: corticícolas (Co), epíxilas (Epx), epifilas (epf), rupícolas (Ru) (Robbins 1952), utilizando-se cupinzeiro $(\mathrm{Cz})$ no lugar de terrícola, pois detalha melhor este substrato para a área.

Análises - A curva de coletor que avalia a suficiência amostral do inventário florístico foi analisada através da estimativa acumulativa de rarefação de espécies utilizando o programa PAST versão 3.01 (Hammer et al. 2001). E a estimativa de riqueza a partir do método de permutação Jackknife pelo programa EstimateS versão 9.1 (Colwell 2013).

A comparação entre as fitofisionomias foi baseada em uma matriz incluindo 18 parcelas usando a presença e ausência das espécies como descritores binários (tabela 1) incluindo as espécies raras. A comparação florística das três fitofisionomias foi calculada através de Análise de Agrupamento pelo Método de Ligação Média não Ponderada (UPGMA) utilizando o índice de similaridade de Jaccard (Margurran 1989), Ordenação Multidimensional Não Métrica (NMDS) utilizando o índice de similaridade de Jaccard, através do programa PAST versão 3.01 (Hammer et al. 2001).

\section{Resultados}

Riqueza - Dentre as 687 amostras de briófitas analisadas, foram encontradas 21 famílias, 57 gêneros e 118 espécies. Sendo 37 espécies de musgos (Bryophyta) (14 famílias e 25 gêneros) e 82 espécies de hepáticas (Marchantiophyta) (sete famílias e 32 gêneros) (tabela 2).

A família de musgos Calymperaceae apresentou a maior riqueza, com oito espécies, seguida de Sematophyllaceae com cinco e, Fissidentaceae, Hypnaceae, Leucobryaceae, Pilotrichaceae, Pterobryaceae e Pylaisiadelphaceae com três espécies cada uma. Calymperaceae e Sematophyllaceae totalizam juntas $10 \%$ de todas as espécies encontradas neste trabalho.

A família de hepáticas Lejeuneaceae apresentou a maior riqueza, com 63 espécies, o que corresponde a $77 \%$ das espécies de hepáticas encontradas.

Foram registradas 19 novas ocorrências de briófitas, sendo 10 ocorrências para o Estado do Pará e nove para a região norte brasileira (tabela 2 ). Cinco espécies não apresentavam as estruturas necessárias para completa caracterização e por serem claramente diferentes das demais encontradas em literatura foram indicadas na listagem florística como morfo-espécies: Cephalozia sp., Ceratolejeunea sp., Lejeunea sp., e Trichosteleum sp.

A suficiência amostral apontou que o número de espécies encontradas deveria seria maior (figura 1) provavelmente devido ao grande número de espécies exclusivas por parcelas (raras na análise), porém a curva de coletor com este número de parcelas já estava estabilizada.

Colonização dos substratos - As 118 espécies encontradas neste levantamento apresentaram a

Tabela 1. Descrição da vegetação e localização nas 35 parcelas amostradas no município de São Domingos do Capim, Estado do Pará, Brasil. *: indica as 18 parcelas utilizadas para comparação.

Table 1. Description and localization of the vegetation of the 35 plots sampled in the São Domingos do Capim municipality, Pará state, Brazil. *: highlight the 18 plots used in the comparison.

\begin{tabular}{lccc}
\hline Fitofisionomia & Área & Coordenadas & Parcelas \\
\hline Floresta de Igapó (IG) & Sítio Santa Joana, igarapé Catita, & $1^{\circ} 50^{\prime} 01,9^{\prime \prime} \mathrm{S}$ & $1^{*}, 2,3^{*}, 4,5,6,7^{*}, 8^{*}, 9,10$, \\
& floresta alagada. & $47^{\circ} 44^{\prime} 57,8^{\prime \prime} \mathrm{W}$ & $21,22,23,24$ \\
Floresta de Igapó (IG) & São Domingos, igarapé Catita, & $1^{\circ} 49^{\prime} 22,9^{\prime \prime} \mathrm{S}$ & $13,16^{*}, 18^{*}, 19,20$ \\
Floresta de Terra Firme & floresta alagada. & $47^{\circ} 42^{\prime} 19,8^{\prime \prime} \mathrm{W}$ & \\
(TE) & São Domingos, igarapé Catita, & $1^{\circ} 49^{\prime} 42^{\prime \prime} \mathrm{S}$ & $11^{*}, 12^{*}, 14^{*}, 15^{*}, 17^{*}, 26^{*}$ \\
Floresta de Várzea, rio & floresta não alagada. & $47^{\circ} 42^{\prime} 14,9^{\prime \prime} \mathrm{W}$ & $1^{*}, 27,28^{*}, 29^{*}, 30^{*}, 31,32$, \\
Capim (VA) & Ilha Assunção, floresta alagada & $1^{\circ} 49^{\prime} 30,1^{\prime \prime S}$ & $25^{*}, 27,33^{*}, 34,35^{*}$ \\
\hline
\end{tabular}


Tabela 2. Espécies de briófitas encontradas e descritores binários de ocorrência nas fitofisionomias e substratos (Fitofisionomia - IG: Floresta de Igapó, VA: Floresta de Várzea, TF: Floresta de Terra Firme; Substrato - Co: corticícola, Epf: epifila, Epx: epíxila, Ru: rupícola, Cz: cupinzeiro; *: indica primeira ocorrência para o Estado do Pará, **: indica primeira ocorrência para a Região Norte).

Table 2. Checklist of the bryophytes and the binary descriptors of the occurrence in the phytophysiognomies and substrates (Phytophysiognomies - IG: Igapó Forest, VA: Várzea Forest, TF: Terra Firme Forest; Substrates - Co: Bark, Epf: epiphyllum, Epx: saxicolous, Ru: terricolous, Cz: termite nest; *: highlight the first record to Pará state, **: highlight the first record to North Region).

\begin{tabular}{|c|c|c|c|c|c|c|c|c|c|}
\hline Família/Espécie & IG & VA & $\mathrm{TF}$ & Co & Epf & Epx & $\mathrm{Ru}$ & $\mathrm{Cz}$ & Voucher \\
\hline \multicolumn{10}{|l|}{ Bryophyta } \\
\hline \multicolumn{10}{|l|}{ Brachytheciaceae } \\
\hline $\begin{array}{l}\text { Zelometeorium patulum (Hedw.) } \\
\text { Manuel }\end{array}$ & 1 & 0 & 1 & 1 & 1 & 1 & 0 & 0 & $163,298,377,389,400$ \\
\hline \multicolumn{10}{|l|}{ Calymperaceae } \\
\hline Calymperes afzelii Sw. & 0 & 1 & 0 & 0 & 0 & 1 & 0 & 0 & 726 \\
\hline Calymperes erosum Müll. Hal. & 1 & 1 & 1 & 1 & 0 & 1 & 0 & 1 & 70 pp., 566, 571, 731 pp. \\
\hline Calymperes levyanum Besch. & 1 & 0 & 1 & 1 & 0 & 1 & 0 & 0 & 425 pp., 386 pp. \\
\hline $\begin{array}{l}\text { Calymperes lonchophyllum } \\
\text { Schwägr. }\end{array}$ & 1 & 0 & 1 & 1 & 0 & 1 & 0 & 0 & 122 pp., 385, 554, 565 pp. \\
\hline Calymperes palisotii Schwägr. & 0 & 1 & 0 & 1 & 0 & 1 & 0 & 0 & 622 pp., 625 pp., 724 pp. \\
\hline Octoblepharum albidum Hedw. & 1 & 1 & 1 & 1 & 0 & 1 & 1 & 1 & $343,352,358,392$ pp. \\
\hline $\begin{array}{l}\text { * Syrrhopodon gardneri (Hook.) } \\
\text { Schwägr. }\end{array}$ & 1 & 0 & 0 & 1 & 0 & 0 & 0 & 0 & $252 \mathrm{pp}$. \\
\hline Syrrhopodon incompletus Schwägr. & 1 & 0 & 1 & 1 & 0 & 1 & 0 & 0 & 413 pp., 422 pp. \\
\hline \multicolumn{10}{|l|}{ Fissidentaceae } \\
\hline Fissidens guianensis Mont. & 1 & 0 & 1 & 1 & 0 & 0 & 1 & 0 & 282,309 pp. \\
\hline *Fissidens inaequalis Mitt. & 1 & 0 & 0 & 1 & 0 & 1 & 0 & 0 & 149,359 pp., 445 pp., 554 \\
\hline *Fissidens ramicola Broth. & 1 & 1 & 1 & 1 & 0 & 1 & 1 & 0 & 86 pp., 314 pp., 573, 645 \\
\hline \multicolumn{10}{|l|}{ Hookeriaceae } \\
\hline $\begin{array}{l}\text { Crossomitrium patrisiae (Brid.) } \\
\text { Müll. Hal. }\end{array}$ & 1 & 0 & 1 & 1 & 1 & 0 & 0 & 0 & 269,331 pp., 399, 446 pp \\
\hline
\end{tabular}

Hypnaceae

Chryso-hypnum diminutivum

(Hampe) W.R. Buck

177 pp., 227, 417, 553

Rhacopilopsis trinitensis (Müll.

Hal.) E. Britton \& Dixon

Vesicularia vesicularis (Schwägr.)

Broth.

$\begin{array}{llllllllll}1 & 0 & 1 & 1 & 0 & 0 & 0 & 0 & 92 \text { pp., } 252 \text { pp., 518, } 611 \text { pp. }\end{array}$

$\begin{array}{lllllllll}1 & 0 & 0 & 0 & 0 & 1 & 0 & 0 & 120 \text { pp., } 236 \text { pp., } 501\end{array}$

Leucobryaceae

Leucobryum laevifolium Broth.

Leucobryum martianum (Hornsch.) Hampe ex Müll. Hal.

Ochrobryum subobtusifolium Broth.

Leucomiaceae

Leucomium strumosum (Hornsch.) Mitt.

$\begin{array}{llllllllll}1 & 0 & 1 & 1 & 0 & 1 & 0 & 0 & 130 \text { pp., } 148 \text { pp., } 157 \text { pp. }\end{array}$

$\begin{array}{llllllllll}1 & 0 & 1 & 1 & 0 & 1 & 1 & 0 & 135 \text { pp., 427, 596, } 597 \text { pp. }\end{array}$

$\begin{array}{lllllllll}1 & 0 & 0 & 1 & 0 & 0 & 0 & 0 & 167 \mathrm{pp} .\end{array}$

Neckeraceae

Neckeropsis undulata (Hedw.)

Reichardt

$\begin{array}{lllllllll}1 & 0 & 1 & 1 & 0 & 1 & 1 & 0 & 90 \text { pp., 361, 440, } 543 \text { pp. }\end{array}$

$\begin{array}{lllllllll}1 & 0 & 0 & 1 & 0 & 1 & 0 & 0 & 85 \text { pp., } 219 \text { pp., } 525 \text { pp. }\end{array}$ 
Tabela 2 (continuação)

Família/Espécie

IG VA TF Co Epf

Epx

$\mathrm{Ru} \mathrm{Cz}$

Voucher

Pilotrichaceae

Callicostella pallida (Hornsch.)

Ångström

$\begin{array}{llllllllll}1 & 0 & 1 & 1 & 0 & 1 & 0 & 0 & 66,471,559 \text { pp., } 581 \text { pp. }\end{array}$

**Lepidopilidium portoricense (Müll. Hal.) (Müll. Hal.) H.A. Crum \& Steere

$\begin{array}{llllllllll}1 & 0 & 0 & 1 & 0 & 1 & 0 & 0 & 140 \text { pp., 267, } 544 \text { pp., } 564\end{array}$

Pilotrichum bipinnatum (Schwägr.) Brid.

$\begin{array}{lllllllll}1 & 0 & 1 & 1 & 0 & 1 & 0 & 0 & 125 \text { pp., } 386 \text { pp., } 525 \text { pp. }\end{array}$

Pterobryaceae

Henicodium geniculatum (Mitt.) W.R.

Buck

$\begin{array}{llllllll}1 & 0 & 0 & 0 & 0 & 1 & 0 & 0\end{array}$

234 pp.

Orthostichopsis tetragona (Hedw.)

Broth.

$\begin{array}{llllllll}0 & 0 & 1 & 1 & 0 & 0 & 0 & 0\end{array}$

604

*Orthostichopsis tortipilis (Müll.

Hal.) Broth.

$\begin{array}{llllllll}0 & 0 & 1 & 1 & 0 & 0 & 0 & 0\end{array}$

382

Pylaisiadelphaceae

Isopterygium tenerifolium Mitt.

$\begin{array}{llllllll}1 & 0 & 0 & 0 & 0 & 1 & 0 & 0\end{array}$

$478 \mathrm{pp}$.

Taxithelium planum (Brid.) Mitt.

Taxithelium sp.

$\begin{array}{llllllll}1 & 1 & 1 & 1 & 0 & 1 & 0 & 0\end{array}$

64 pp., 112, 316, 729 pp.

$\begin{array}{lllllllll}0 & 0 & 1 & 0 & 0 & 1 & 0 & 0 & 383 \mathrm{pp} .\end{array}$

Sematophyllaceae

Sematophyllum galipense (Müll.

Hal.) Mitt.

Sematophyllum subsimplex (Hedw.)

Mitt.

Trichosteleum sentosum (Sull.) A.

Jaeger

Trichosteleum $\mathrm{sp.}$

Trichosteleum subdemissum (Besch.)

A. Jaeger

$\begin{array}{llllllll}1 & 0 & 0 & 0 & 0 & 1 & 0 & 0\end{array}$

532

Stereophyllaceae

Pilosium chlorophyllum (Hornsch.)

Müll. Hal.

$$
\begin{array}{ccccccccc}
1 & 1 & 1 & 1 & 0 & 1 & 1 & 0 & 92 \text { pp., 329, 568, } 582 \mathrm{pp} . \\
1 & 0 & 0 & 0 & 0 & 1 & 0 & 0 & 208 \mathrm{pp} ., 442,477,559 \mathrm{pp} . \\
0 & 0 & 1 & 0 & 0 & 1 & 0 & 0 & 288,597 \text { pp., } 603 \mathrm{pp} . \\
1 & 1 & 0 & 1 & 0 & 1 & 0 & 0 & 63,444,569 \mathrm{pp} ., 588 \mathrm{pp} .
\end{array}
$$

Thuidiaceae

Pelekium schistocalyx (Müll.Hal.)

Touw

$\begin{array}{lllllllll}1 & 0 & 0 & 1 & 0 & 1 & 0 & 0 & 110,358 \text { pp., } 563\end{array}$

Marchantiophyta

Aneuraceae

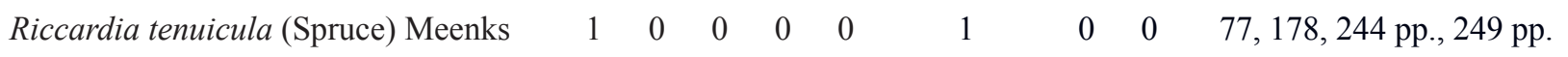

Cephaloziaceae

Cephalozia sp.

Lejeuneaceae

$\begin{array}{llllllllllll}\text { Acrolejeunea emergens (Mitt.) Steph. } & 1 & 1 & 0 & 1 & 0 & 0 & 0 & 0 & 365 \text { pp., } 622 \text { pp., } 625 \text { pp. }\end{array}$

Archilejeunea auberiana (Mont.) A.

Evans

$\begin{array}{lllllllll}1 & 1 & 0 & 1 & 0 & 1 & 0 & 0 & 72,198 \text { pp., } 206 \text { pp., } 733\end{array}$


Tabela 2 (continuação)

\begin{tabular}{|c|c|c|c|c|c|c|c|c|c|}
\hline Família/Espécie & IG & VA & $\mathrm{TF}$ & Co & Epf & Epx & $\mathrm{Ru}$ & $\mathrm{Cz}$ & Voucher \\
\hline $\begin{array}{l}\text { Archilejeunea fuscescens (Hampe ex } \\
\text { Lehm.) Fulford }\end{array}$ & 1 & 1 & 0 & 1 & 0 & 0 & 0 & 0 & 134 pp., 252 pp., 295 pp. \\
\hline $\begin{array}{l}\text { Archilejeunea parviflora (Nees) } \\
\text { Schiffn. }\end{array}$ & 1 & 1 & 1 & 1 & 0 & 0 & 0 & 0 & $313,320,369,613$ \\
\hline $\begin{array}{l}\text { Caudalejeunea lehmanniana } \\
\text { (Gottsche) A. Evans }\end{array}$ & 1 & 0 & 0 & 1 & 0 & 0 & 0 & 0 & $420 \mathrm{pp}$ \\
\hline $\begin{array}{l}\text { Ceratolejeunea coarina (Gottsche) } \\
\text { Steph. }\end{array}$ & 1 & 1 & 1 & 1 & 1 & 1 & 0 & 0 & $165,238,439,740 \mathrm{pp}$. \\
\hline $\begin{array}{l}\text { Ceratolejeunea cornuta (Lindenb.) } \\
\text { Schiffn. }\end{array}$ & 1 & 1 & 0 & 1 & 0 & 1 & 0 & 0 & 78 pp., 623, 685, 739 pp. \\
\hline $\begin{array}{l}\text { Ceratolejeunea dussiana (Steph.) G. } \\
\text { Dauphin }\end{array}$ & 1 & 0 & 0 & 1 & 0 & 0 & 0 & 0 & $164 \mathrm{pp}$. \\
\hline $\begin{array}{l}\text { Ceratolejeunea guianensis (Nees \& } \\
\text { Mont.) Steph. }\end{array}$ & 1 & 1 & 0 & 1 & 0 & 0 & 0 & 0 & 512, 642 pp., 707 pp., 735 \\
\hline $\begin{array}{l}\text { Ceratolejeunea laetefusca (Austin) } \\
\text { R.M.Schust. }\end{array}$ & 1 & 1 & 0 & 1 & 0 & 0 & 0 & 0 & 654 pp., 709 pp., 731 pp. \\
\hline Ceratolejeunea minuta Dauphin & 1 & 1 & 1 & 1 & 0 & 0 & 0 & 0 & 102 pp., 487, 510, 606 \\
\hline Ceratolejeunea sp. & 0 & 1 & 0 & 1 & 0 & 0 & 0 & 0 & $621,629,713$ \\
\hline $\begin{array}{l}\text { Cheilolejeunea clausa(Nees \& } \\
\text { Mont.) R.M. Schust. }\end{array}$ & 1 & 1 & 0 & 1 & 0 & 0 & 0 & 0 & 418 pp., 650 pp., 652 pp. \\
\hline $\begin{array}{l}\text { **Cheilolejeunea comans (Spruce) } \\
\text { R.M. Schust. }\end{array}$ & 0 & 1 & 0 & 1 & 0 & 0 & 0 & 0 & $731 \mathrm{pp}$. \\
\hline $\begin{array}{l}\text { **Cheilolejeunea discoidea (Lehm. \& } \\
\text { Lindenb.) Kachr. \& R.M. Schust. }\end{array}$ & 0 & 1 & 0 & 1 & 0 & 0 & 0 & 0 & 633 \\
\hline $\begin{array}{l}\text { Cheilolejeunea holostipa (Spruce) } \\
\text { Grolle \& R.-L. Zhu }\end{array}$ & 0 & 1 & 0 & 1 & 0 & 0 & 0 & 0 & $717 \mathrm{pp}$. \\
\hline $\begin{array}{l}\text { *Cheilolejeunea neblinensis Ilkiu- } \\
\text { Borges \& Gradst. }\end{array}$ & 1 & 0 & 0 & 1 & 0 & 0 & 0 & 0 & $497 \mathrm{pp}$. \\
\hline $\begin{array}{l}\text { Cheilolejeunea rigidula (Mont.) R.M. } \\
\text { Schust. }\end{array}$ & 1 & 1 & 1 & 1 & 0 & 1 & 0 & 0 & 179 pp., 194, 649, 737 \\
\hline $\begin{array}{l}\text { Cheilolejeunea trifaria (Reinw. et al.) } \\
\text { Mizut. }\end{array}$ & 1 & 0 & 0 & 1 & 0 & 1 & 0 & 0 & 69 pp., 71 \\
\hline $\begin{array}{l}\text { Cololejeunea camilli (Lehm.) A. } \\
\text { Evans }\end{array}$ & 0 & 1 & 0 & 0 & 0 & 1 & 0 & 0 & 659 pp. \\
\hline Cololejeunea contractiloba A. Evans & 1 & 0 & 0 & 1 & 0 & 0 & 0 & 0 & 159 pp., 259 pp., 300 pp. \\
\hline $\begin{array}{l}\text { Cololejeunea gracilis (Jovet-Ast) } \\
\text { Pócs }\end{array}$ & 1 & 1 & 0 & 1 & 0 & 1 & 0 & 0 & $516 \mathrm{pp}, 658$ \\
\hline $\begin{array}{l}\text { ** Cololejeunea microscópica } \\
\text { (Taylor) Schiffn. }\end{array}$ & 1 & 1 & 0 & 1 & 0 & 1 & 0 & 0 & 484 pp., 586, 727 \\
\hline $\begin{array}{l}\text { Cololejeunea truncatifolia (Horik.) } \\
\text { Mizut. }\end{array}$ & 1 & 1 & 1 & 1 & 1 & 1 & 0 & 0 & 116 pp., 379, 460, 656 pp. \\
\hline $\begin{array}{l}\text { Cololejeunea winkleri (M. I. Morales } \\
\text { Z. \& A. Lücking) Bernecker \& Pócs }\end{array}$ & 1 & 1 & 1 & 1 & 1 & 0 & 0 & 0 & 373 pp., 413 pp., 647 pp. \\
\hline $\begin{array}{l}\text { Cyclolejeunea convexistipa (Lehm. \& } \\
\text { Lindenb.) A. Evans }\end{array}$ & 1 & 0 & 0 & 1 & 1 & 0 & 0 & 0 & 261 pp., 309 pp., 498 pp. \\
\hline
\end{tabular}


Tabela 2 (continuação)

\begin{tabular}{|c|c|c|c|c|c|c|c|c|c|}
\hline Família/Espécie & IG & VA & $\mathrm{TF}$ & Co & Epf & Epx & $\mathrm{Ru}$ & $\mathrm{Cz}$ & Voucher \\
\hline Diplasiolejeunea brunnea Steph. & 1 & 0 & 1 & 0 & 1 & 0 & 0 & 0 & 261 pp., 598 pp. \\
\hline $\begin{array}{l}\text { Haplolejeunea cucullata (Steph.) } \\
\text { Grolle }\end{array}$ & 0 & 0 & 1 & 1 & 0 & 1 & 0 & 0 & 286,287 \\
\hline $\begin{array}{l}\text { Harpalejeunea oxyphylla (Nees \& } \\
\text { Mont.) Stephani }\end{array}$ & 1 & 0 & 0 & 1 & 0 & 0 & 0 & 0 & 246 pp., 265 pp. \\
\hline $\begin{array}{l}\text { Harpalejeunea stricta (Lindenb. \& } \\
\text { Gottsche) Steph. }\end{array}$ & 0 & 1 & 0 & 1 & 0 & 0 & 0 & 0 & 644 pp., 704 pp., 715 pp. \\
\hline Lejeunea asperrima Spruce & 1 & 0 & 0 & 1 & 0 & 0 & 0 & 0 & 370 pp., 372a pp., 449 pp. \\
\hline Lejeunea boryana Mont. & 1 & 0 & 0 & 1 & 0 & 0 & 0 & 0 & 115 pp., 570 \\
\hline $\begin{array}{l}\text { Lejeunea caespitosa Lindenb. ex } \\
\text { Gott. }\end{array}$ & 1 & 0 & 0 & 1 & 0 & 0 & 0 & 0 & 62 pp., 493 \\
\hline Lejeunea calcicola R.M. Schust. & 1 & 0 & 0 & 0 & 0 & 1 & 0 & 0 & 511 \\
\hline $\begin{array}{l}\text { *Lejeunea cerina (Lehm. \& Lindenb.) } \\
\text { Gottsche }\end{array}$ & 1 & 0 & 1 & 1 & 0 & 1 & 1 & 0 & 67 pp., 139, 314 pp., 539 \\
\hline Lejeunea controversa Gottsche & 1 & 0 & 1 & 1 & 0 & 1 & 1 & 0 & 129 pp., 280 pp., 514 pp. \\
\hline **Lejeunea filipes Spruce & 1 & 1 & 0 & 1 & 0 & 1 & 0 & 0 & 419 pp., 668 pp. \\
\hline Lejeunea flava (Sw.) Nees & 1 & 1 & 0 & 1 & 0 & 1 & 0 & 0 & $585,694,714,716 \mathrm{pp}$. \\
\hline **Lejeunea immersa Spruce & 1 & 0 & 0 & 1 & 0 & 0 & 0 & 0 & $76,90 \mathrm{pp}$ \\
\hline Lejeunea laetevirens Nees \& Mont. & 1 & 1 & 0 & 1 & 0 & 0 & 0 & 0 & 203, 376 pp., 641 pp. \\
\hline Lejeunea minutiloba A. Evans & 1 & 0 & 0 & 0 & 0 & 1 & 0 & 0 & $131 \mathrm{pp}$ \\
\hline **Lejeunea raddiana Lindenb. & 1 & 0 & 0 & 1 & 0 & 0 & 0 & 0 & 250 \\
\hline Lejeunea sp. & 1 & 1 & 0 & 0 & 0 & 1 & 0 & 0 & 127 pp., 659 pp., 668 pp. \\
\hline Lejeunea tapajosensis Spruce & 1 & 1 & 1 & 1 & 0 & 1 & 0 & 0 & $705,732 \mathrm{pp}$ \\
\hline $\begin{array}{l}\text { Lopholejeunea nigricans (Lindenb.) } \\
\text { Schiffn. }\end{array}$ & 1 & 1 & 0 & 1 & 0 & 1 & 0 & 0 & 293 pp., 612 pp., 657 pp. \\
\hline $\begin{array}{l}\text { Lopholejeunea subfusca (Nees) } \\
\text { Schiffn. }\end{array}$ & 0 & 1 & 1 & 1 & 0 & 0 & 0 & 0 & 620 pp., 651 pp., 710 pp. \\
\hline $\begin{array}{l}\text { Mastigolejeunea auriculata (Wilson) } \\
\text { Schiffn. }\end{array}$ & 1 & 1 & 1 & 1 & 0 & 1 & 0 & 0 & 234 pp., 626 pp., 702 pp. \\
\hline Microlejeunea epiphylla Bischl. & 0 & 1 & 0 & 1 & 0 & 0 & 0 & 0 & 689 pp., 709 pp. \\
\hline Microlejeunea ruthii A. Evans & 0 & 1 & 0 & 1 & 0 & 0 & 0 & 0 & 689 pp. \\
\hline $\begin{array}{l}\text { Odontolejeunea lunulata (Weber) } \\
\text { Schiffn. }\end{array}$ & 1 & 0 & 0 & 0 & 1 & 0 & 0 & 0 & 488 \\
\hline $\begin{array}{l}\text { Pictolejeunea picta(Gottsche ex } \\
\text { Steph.) Grolle }\end{array}$ & 1 & 0 & 1 & 1 & 0 & 1 & 0 & 0 & $118,245,392$ pp., 574 pp. \\
\hline $\begin{array}{l}\text { Prionolejeunea muricato-serrulata } \\
\text { (Spruce) Steph. }\end{array}$ & 1 & 1 & 1 & 1 & 0 & 1 & 1 & 0 & 193, 309 pp., 646 pp. \\
\hline $\begin{array}{l}\text { Pycnolejeunea contigua (Nees) } \\
\text { Grolle }\end{array}$ & 0 & 1 & 0 & 1 & 0 & 0 & 0 & 0 & $660 \mathrm{pp}$. \\
\hline $\begin{array}{l}\text { *Rectolejeunea berteroana (Gottsche } \\
\text { ex Steph.) A. Evans }\end{array}$ & 0 & 1 & 0 & 1 & 0 & 0 & 0 & 0 & 709 pp., 637 \\
\hline $\begin{array}{l}\text { Schiffneriolejeunea polycarpa (Nees) } \\
\text { Gradst. }\end{array}$ & 0 & 1 & 0 & 1 & 0 & 0 & 0 & 0 & $698 \mathrm{pp}$ \\
\hline $\begin{array}{l}\text { Stictolejeunea squamata (Willd. ex } \\
\text { Weber) Schiffn. }\end{array}$ & 1 & 1 & 1 & 1 & 0 & 1 & 0 & 0 & 82, 198 pp., 524, 731 pp. \\
\hline
\end{tabular}


Tabela 2 (continuação)

\begin{tabular}{|c|c|c|c|c|c|c|c|c|c|}
\hline Família/Espécie & IG & VA & $\mathrm{TF}$ & Co & Epf & Epx & $\mathrm{Ru}$ & $\mathrm{Cz}$ & Voucher \\
\hline $\begin{array}{l}\text { Symbiezidium barbiflorum (Lindenb. } \\
\text { \& Gottsche) A. Evans }\end{array}$ & 1 & 1 & 0 & 1 & 0 & 0 & 0 & 0 & 103, 235 pp., 647 pp., 684 \\
\hline Symbiezidium transversale (Sw.) Trevis. & 1 & 1 & 1 & 1 & 0 & 1 & 0 & 0 & 70 pp., 500, 690, 740 pp. \\
\hline $\begin{array}{l}\text { Taxilejeunea obtusangula(Spruce) A. } \\
\text { Evans }\end{array}$ & 1 & 0 & 0 & 1 & 0 & 0 & 0 & 0 & $297 \mathrm{pp}$. \\
\hline $\begin{array}{l}\text { *Trachylejeunea aneogyna (Spruce) } \\
\text { Grolle }\end{array}$ & 0 & 1 & 0 & 0 & 0 & 1 & 0 & 0 & $612 \mathrm{pp}$. \\
\hline $\begin{array}{l}\text { Trachylejeunea pandurantha } \\
\text { (Spruce) Steph. }\end{array}$ & 1 & 0 & 1 & 1 & 0 & 1 & 1 & 0 & 65 pp., 214, 575, 603 pp. \\
\hline $\begin{array}{l}\text { **Vitalianthus bischlerianus (Porto \& } \\
\text { Grolle) R.M. Schust. \& Giancotti }\end{array}$ & 1 & 1 & 1 & 1 & 0 & 0 & 0 & 0 & 289 pp., 497 pp., 537 pp. \\
\hline $\begin{array}{l}\text { Xylolejeune acrenata (Nees\& Mont.) } \\
\text { X.-L. He \&Grolle }\end{array}$ & 1 & 0 & 0 & 0 & 0 & 1 & 0 & 0 & $131 \mathrm{pp}$. \\
\hline \multicolumn{10}{|l|}{ Lepidoziaceae } \\
\hline $\begin{array}{l}\text { Zoopsidella macella (Steph.) R.M. } \\
\text { Schust. }\end{array}$ & 1 & 0 & 1 & 0 & 0 & 1 & 0 & 0 & 366,390 pp., 391 pp. \\
\hline \multicolumn{10}{|l|}{ Lophocoleaceae } \\
\hline $\begin{array}{l}\text { Chiloscyphus liebmannianus (Gottsche) } \\
\text { J.J. Engel \& R.M. Schust. }\end{array}$ & 1 & 0 & 0 & 1 & 0 & 1 & 0 & 0 & 89 pp., 102 pp., 190 pp. \\
\hline $\begin{array}{l}\text { Chiloscyphus martianus (Nees) J.J. } \\
\text { Engel \& R.M. Schust. }\end{array}$ & 1 & 0 & 0 & 1 & 0 & 0 & 0 & 0 & 557 \\
\hline $\begin{array}{l}\text { Chiloscyphus perissodontus (Spruce) } \\
\text { J.J. Engel \& R.M. Schust. }\end{array}$ & 1 & 0 & 1 & 1 & 0 & 1 & 0 & 0 & 96 pp., 130 pp., 367 pp. \\
\hline \multicolumn{10}{|l|}{ Plagiochilaceae } \\
\hline $\begin{array}{l}\text { *Plagiochila corrugata(Nees) Nees \& } \\
\text { Mont. }\end{array}$ & 1 & 1 & 0 & 1 & 0 & 1 & 0 & 0 & 73 pp., 422 pp., 703 pp. \\
\hline $\begin{array}{l}\text { Plagiochila disticha (Lehm. \& } \\
\text { Lindenb.) Lindenb. }\end{array}$ & 1 & 1 & 1 & 1 & 0 & 1 & 0 & 0 & 95 pp., 260, 429, 720 pp. \\
\hline Plagiochila macrostachya Lindenb. & 1 & 1 & 1 & 1 & 0 & 1 & 0 & 0 & 107 pp., $255,368,675$ pp \\
\hline Plagiochila martiana (Nees) Lindenb. & 0 & 0 & 1 & 1 & 0 & 0 & 0 & 0 & 273 \\
\hline **Plagiochila patentíssima Lindenb. & 1 & 0 & 1 & 1 & 0 & 0 & 0 & 0 & 81 pp., $196,447,508$ pp. \\
\hline Plagiochila rutilans Lindenb. & 1 & 0 & 1 & 1 & 0 & 0 & 0 & 0 & 173 pp., 252 pp., 458 pp. \\
\hline Plagiochila simplex (Sw.) Lindenb. & 1 & 0 & 0 & 1 & 0 & 0 & 0 & 0 & 210 pp. \\
\hline Plagiochila subplana Lindenb. & 1 & 0 & 1 & 1 & 0 & 1 & 0 & 0 & 97 pp., 222, 300 pp. \\
\hline \multicolumn{10}{|l|}{ Radulaceae } \\
\hline Radula flaccida Lindenb. \& Gottsche & 1 & 0 & 1 & 1 & 1 & 0 & 0 & 0 & 100 pp., 344, 388, 542 pp. \\
\hline Radula javanica Gottsche & 1 & 1 & 1 & 1 & 0 & 1 & 0 & 0 & 179 pp., 279, 317, 595 pp. \\
\hline *Radula mammosa Spruce & 1 & 0 & 0 & 1 & 0 & 0 & 0 & 0 & 62 pp., 107 pp., 258 pp. \\
\hline Radula quadrata Gottsche & 0 & 0 & 1 & 1 & 0 & 0 & 0 & 0 & 290 \\
\hline Radula recubans Taylor & 1 & 0 & 0 & 1 & 0 & 0 & 0 & 0 & 309 pp. \\
\hline Total & 97 & 52 & 53 & 101 & 9 & 66 & 12 & 2 & \\
\hline
\end{tabular}


seguinte distribuição segundo a colonização de substratos: Co $(85 \%)>\operatorname{Epx}(55 \%)>\mathrm{Ru}(10 \%)>\operatorname{Epf}$ $(7,5 \%)>\mathrm{Cz}(2 \%)$ (figura 2$)$.

Foi observada especificidade de substrato por parte da brioflora, sendo que 63 espécies $(53 \%$ das ocorrências) foram exclusivas de um substrato e 56 (47\%) foram generalistas (figura 2), ou seja, não apresentaram especificidade de substrato (destas, 42 (35\%) ocorreram em dois substratos (Co, Epx), 13 espécies (11\%) em três substratos (Co, Epx, Epf) e, somente Octoblepharum albidum ocorreu em quatro substratos (Co, Epx, $\mathrm{Ru}, \mathrm{Cz})$.

As hepáticas tiveram o maior número de ocorrências exclusivas como corticícolas $(34 \%, 40$ espécies), seguida de epíxilas (6,5\%, oito espécies) e epifilas $(1,5 \%$, duas espécies, Diplasiolejeunea brunnea e Odontolejeunea lunulata, ambas da família Lejeuneaceae). As famílias com maior número de espécies exclusivamente corticícolas foram Lejeuneaceae, Plagiochilaceae, Radulaceae e Lophocoleaceae, e as famílias mais frequentes com espécies epíxilas foram Lejeuneaceae, Aneuraceae e Lepidoziaceae.

Os musgos tiveram maior número de ocorrências exclusivas como epíxilas $(6,5 \%$, oito espécies $)$ e corticícolas $(4,2 \%$, cinco espécies).

Distribuição da comunidade de briófitas entre as fitofisionomias - Na comparação entre as três fitofisionomias a maior riqueza de briófitas foi registrada na IG, com 97 espécies (o que corresponde a $81 \%$ do total), seguida de TE com 53 (44\%) e VA com 52 (43\%) (figura 3).

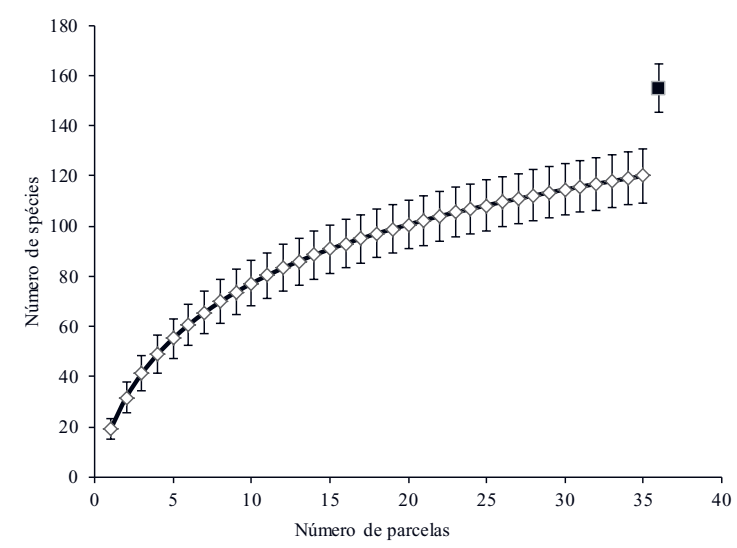

Figura 1. Estimativa de riqueza a partir do método de permutação Jackknife mostrando que a riqueza observada está abaixo da esperada. $\_$Riqueza observada, $\leadsto-$ Jackknife.

Figure 1. Richness estimation by the Jackknife permutation, showing that the richness is below than expected, $\longrightarrow$ Richness obtened, -₫- Jackknife.
Exceto por uma área de TF a ordenação (UPGMA) e agrupamento (NMDS) mostram agrupamento entre as parcelas das fitofisionomias, sendo entre IG e TF (figura 4) mais próximas entre si (figura 5).

A riqueza de musgos foi maior na IG com 31 espécies (26\%), seguido de TE com 22 (18\%) e VA com oito (7\%). Cinco das espécies encontradas neste levantamento foram comuns às três fitofisionomias analisadas: Calymperes erosum, Octoblepharum albidum, Fissidens ramicola, Sematophyllum subsimplex e Taxithelium planum.

A riqueza de hepáticas foi maior em IG com 66 (55\%), seguida por VA com $44(36 \%)$ e TE com $31(26 \%)$. Quinze espécies foram comuns às três fitofisionomias analisadas: Aphanolejeunea truncatifolia, Aphanolejeunea winkleri, Archilejeunea parviflora, Ceratolejeunea coarina, Ceratolejeunea minuta, Cheilolejeunea rigidula, Lejeunea tapajosensis, Mastigolejeunea auriculata, Prionolejeunea muricato-serrulata, Stictolejeunea squamata, Symbiezidium transversale, Vitalianthus bischlerianus, Plagiochila disticha, Plagiochila macrostachya e Radula javanica.

A colonização dos substratos entre as três fitofisionomias seguiu este padrão: $\mathrm{Co}>\mathrm{Epx}>\mathrm{Ru}>\mathrm{Epf}>\mathrm{Cz}$, porém, a IG apresentou maior número de espécies para o substrato corticícola.

\section{Discussão}

Riqueza - A riqueza de espécies, gêneros e famílias encontrada na listagem florística está próximo às 120 espécies, 59 gêneros e 23 famílias (79 hepáticas, 66\% do total) encontradas por Alvarenga \& Lisboa (2009) para a Amazônia Oriental e as 109 espécies, 52 gêneros e 20 famílias (77 hepáticas, 71\%) encontradas por Benavides et al. (2004) para a Amazônia Ocidental.

A grande quantidade de espécies de Lejeuneaceae encontradas está de acordo com o que Gradstein \& Costa (2003) relatam sobre a riqueza dessa família para a Amazônia, sendo um dos táxons mais importantes de hepáticas folhosas para esse domínio. O número de espécies de hepáticas que ocorre na Amazônia corresponde a 27,4\% das espécies deste domínio fitogeográfico, sendo o segundo maior em diversidade de hepáticas do Brasil (Gradstein \& Costa 2003) e aproximadamente 3\% das que ocorrem na América tropical de acordo com Gradstein et al. (2001).

O número de espécies da família Lejeuneaceae encontrado neste levantamento corresponde a $91,3 \%$ 
do total de espécies de hepáticas até agora registradas para o Estado do Pará e está próximo do número de espécies encontrado na Estação Científica Ferreira Penna (57 espécies) por Ilkiu-Borges (2000), o que mostra que a estimativa de riqueza está próxima para o ocorrente na região.

De acordo com Gradstein et al. (2001) as famílias de musgos Calymperaceae, Sematophyllaceae,

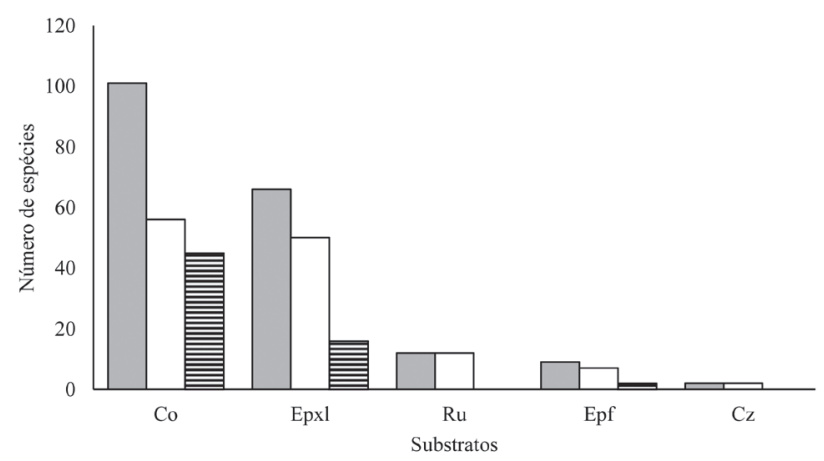

Figura 2. Distribuição das espécies de briófitas encontradas em relação ao substrato colonizado, comparando a ocorrência total, comuns e exclusivas (Co: corticícola, Epx: epíxila, $\mathrm{Ru}$ : rupícola, Epf: epifila e Cz: cupinzeiro). $\equiv$ Total, $\square$ Comuns, Exclusivas.

Figure 2. Distribution of bryophytes species in substrate, comparing the occurrence of total, frequent and exclusive (Co: bark, Epx: saxicolous, Ru: rock, Epf: epiphyllum and $\mathrm{Cz}$ : termite nest). Total, $\square$ Frequent, $\square$ Exclusive.

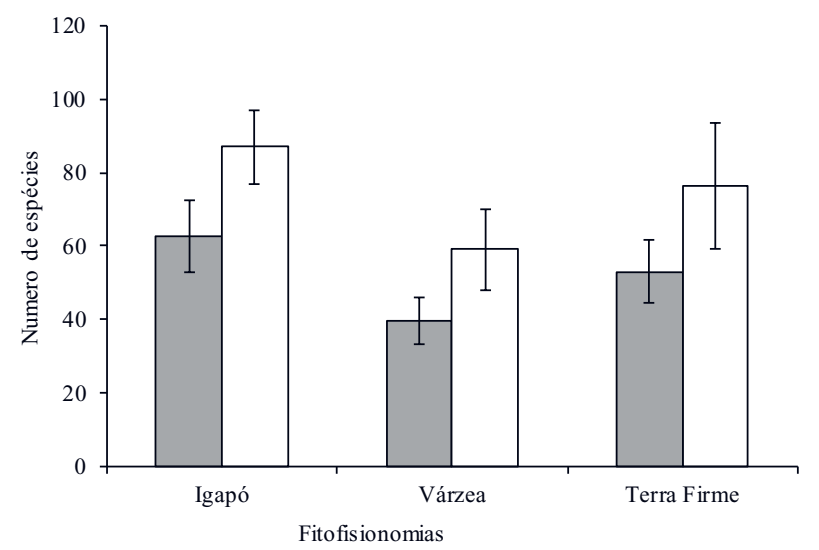

Figura 3 - Distribuição das espécies de briófitas encontradas em relação às fitofisionomias, comparando as estimativas obtidas por Jackknife (intervalo de confiança $<95 \%$ ). — Riqueza observada, $\square$ Riqueza estimada.

Figure 3. Distribution of bryophytes species in the phytophysiognomies, with the Jackknife estimation (confidence interval $<95 \%$ ). Richness obteined, $\square$ Richness estimated.
Fissidentaceae e Pilotrichaceae correspondem a 50\% do total da diversidade de musgos na Amazônia e estas, também foram as mais ricas neste levantamento.

Colonização dos substratos - A maioria das espécies de briófitas que ocorrem nas florestas tropicais está relacionada com vegetação ocorrente, pois dependem da sua disponibilidade como substrato (Ruinen 1953). Isso está de acordo com os resultados encontrados, tendo em vista que $54 \%$ das espécies registradas ocorrem em troncos, vivos ou mortos, e em folhas que são os substratos mais abundantes na área estudada.

A ocorrência das duas espécies de Calymperaceae sobre cupinzeiro reafirma a grande amplitude ecológica desta família na Amazônia descrita em Gradstein et al. (2001).

A espécie Diplasiolejeunea brunnea encontrada neste levantamento somente como epifila, apresenta menor amplitude ecológica que a descrita por IlkiuBorges (2000) para Estação Científica Ferreira Penna, Caxiuanã onde ela foi registrada como corticícola, o que pode indicar que a área é bem preservada.

A ausência de espécies terrícolas segue a ausência de espécies no solo de florestas causado pelo acúmulo e constante deposição de folhas como descrito por Wagner et al. (2014). E no caso da VA o solo é constantemente lavado pelo alagamento sazonal e na IG não existe solo disponível para colonização das briófitas.

Existe em literatura a descrição de espécies beneficiadas pelo alagamento em florestas temperadas, como Ephemerum crassinervium, Riccia hirta, Riccia huebeneriana e Ricciocarpus natans (Middleton 2002), e Ephemerum cohaerens e E. rutheanum (Bjlsma et al. 2012), porém o alagamento descrito nestes trabalhos trata de solos saturados e com apenas uma película de água sobre o solo. Uma situação diferente da encontrada em VA e IG onde o nível da água varia de um a oito metros entre as estações do ano.

Distribuição da comunidade de briófitas entre as fitofisionomias - A fitofisionomia mais rica foi IG mesmo apresentando o solo constantemente alagado, o que inviabiliza a colonização deste substrato. A Floresta de Terra Firme foi o segundo ambiente mais rico por apresentar a maior disponibilidade de substrato e a Floresta de Várzea, devido ao seu alagamento sazonal possui a menor riqueza, provavelmente devido ao alagamento inconstante.

De acordo com Proctor (1982) e Wagner et al. (2014) a tolerância às altas temperaturas, à alta radiação luminosa e a à dessecação são as características 
ecofisiológicas chaves que moldam a comunidade de briófitas em ambientes tropicais.

$O$ resultado obtido neste trabalho indica as fitofisionomias IG e TF possuem as floras mais similares e ambas compartilham espécies com VA, mesmo a VA e TF possuindo o solo como substrato colonizável em comum e IG não.

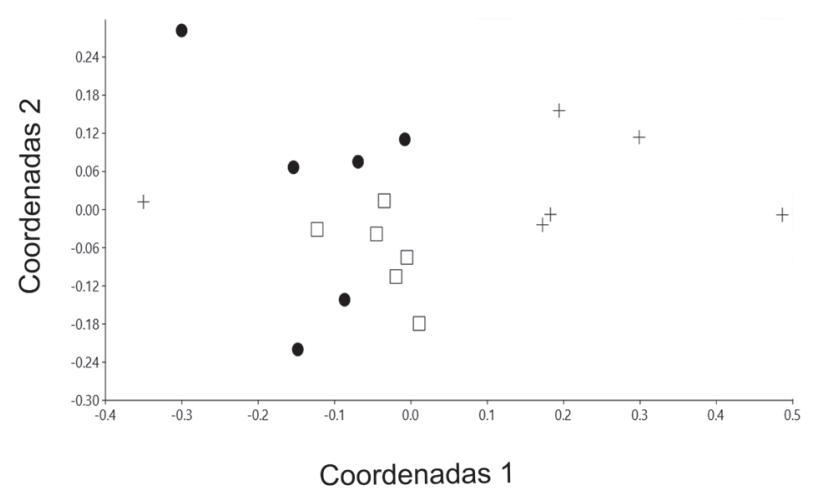

Figura 4. Diagrama produzido pela análise de ordenação multidimensional não métrica (NMDS) da composição florística das 18 parcelas analisadas para a comparação entre as três fitofisionomias através do índice de Similaridade Jaccard. Igapó, • Terra Firme, + Várzea.

Figure 4. Non metric multidimensional ordination (NMDS) of the floristic composition for the 18 plots analyzed to the comparison of the three phytophysiognomies using Jaccard similarity index. $\square$ Igapó,• Terra Firme, + Várzea.

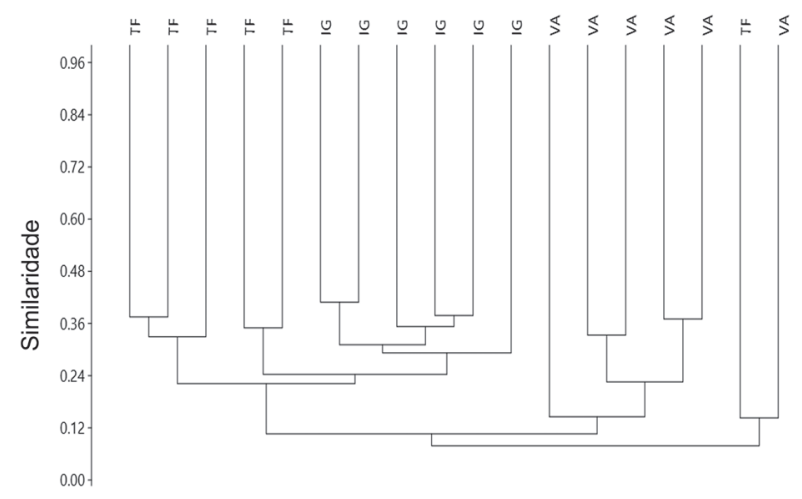

Figura 5. Dendrograma da composição florística das 18 parcelas analisadas para a comparação entre as três fitofisionomias através do índice de similaridade de Jaccard (95\% com coeficiente de correlação cofenética a 0,8306) (IG: Floresta de Igapó, TF: Floresta de Terra Firme, VA: Floresta de Várzea).

Figure 5. Dendrogram of the floristic composition for the 18 plots analyzed to the comparison of the three phytophysiognomies using Jaccard similarity index ( $95 \%$ of cofenetic correlation coefficient with 0,8306) (IG: Igapó Forest, TF: Terra Firme Forest, VA: Várzea Forest).
Táxons com menor tolerância à dessecação são excluídos de ambientes influenciados por regime hídrico sazonal ou inconstante, o que causa especialização em ambientes sujeitos a estresse hídrico (Wagner et al. 2014) e considerados especialistas, e aqueles que possuem maior tolerância a dessecação são generalistas.

Dentre as espécies citadas como tolerantes à dessecação, ou seja, generalistas, e podem indicar que a área está em recuperação estão Calymperes erosum e Sematophyllum subsimplex, encontradas em todas as fitofisionomias deste estudo e também em todas as fitofisionomias estudadas por Moraes (2006) na Estação Científica Ferreira Penna.

O número de espécies encontrado difere do apresentado por Alvarenga \& Lisboa (2009) que apresenta a TE como mais rica, seguida de VA e $\mathrm{IG}$, porém os próprios autores apontam falha na padronização da amostragem. Para esta mesma área, lkiu-Borges (2000), realizando inventário apenas da família Lejeuneaceae, encontrou maior riqueza de espécies para a VA. Porém ambos os trabalhos não apresentam análise de suficiência amostral o que compromete a comparação com os resultados obtidos.

Benavides et al. (2004) encontrou um número de espécies semelhante entre a Mata de Terra Firme e os “ambientes inundáveis" (incluindo VA a IG), o que difere com os resultados encontrados neste levantamento, onde a IG foi a mais rica. Porém, Benavides et al. (2004), apesar de encontrar número semelhante de espécies entre os ambientes estudados, descrevem agrupamento das comunidades de briófitas entre ambientes inundáveis e Terra Firme semelhante ao resultado obtido neste trabalho.

Os resultados obtidos mostram que as fitofisionomias analisadas apresentam diferenças na riqueza (IG $>\mathrm{TF}$ $>$ VA) e composição das comunidades de briófitas, devido à existência de espécies exclusivas e as análises de Agrupamento (UPGMA) e Ordenação (NMDS) indicarem estruturação das comunidades entre as três fitofisionomias, o que enfatiza a necessidade de análises que indiquem os fatores que influenciem nessas diferenças.

\section{Literatura citada}

Alvarenga, L.D.P. \& Lisboa, R.C.L. 2009. Contribuição para o conhecimento da taxonomia, ecologia e fitogeografia de Briófitas da Amazônia Oriental. Acta Amazonica 39: 495-504.

Benavides, J.C., Idarraga, A. \& Aivarez, E. 2004. Bryophyte diversity patterns in flooded and tierra firme forests in the Araracuara Region, Colombian Amazonia. Tropical Bryology 25: 117-126. 
Bjlsma, R.-J., Nieuwkoop, J. \& Siebel, H. 2012. Ephemerum cohaerens and E. rutheanum: persisten annual bryophytes in the Dutch. Lindbergia 35: 63-75.

Buck,W.R. 1998. Pleurocarpous mosses of the West Indies. Memoirs of The New York Botanical Garden 82: 1-400.

Colwell, K. 2013. EstimateS: Statistical Estimations of Species Richness and Shared Species from Samples, versão. 9.1.0. University of Connectiut, USA Copyright, Connectiut.

Costa, D.P. \& Peralta, D.F. 2015. Bryophytes diversity in Brazil. Rodriguésia 66: 1063-1071.

Costa, D.P., Almeida, J.S.S., Dias, N.S., Gradstein, S.R. \& Churchill, S.P. 2010. Manual de Briologia. 1 ed. Editora Interciência, Rio de Janeiro.

Costa, D.P., Pôrto, K.C., Luizi-Ponzo, A.P., Ilkiu-Borges, A.L., Bastos, C.J.P,. Câmara, P.E.A.S., Peralta, D.F., Bôas-Bastos, S.B.V., Imbassahy, C.A.A., Henriques, D.K., Gomes, H.C.S., Rocha, L.M., Santos, N.D., Siviero, T.S., Vaz-Imbassahy, T.D.F. \& Churchill, S.P. 2011. Synopsis of the Brazilian moss flora: checklist, distribution and conservation. Nova Hedwigia 93: 277-334.

Crandall-Stotler, B., Stotler, R.E. \& Long, D.G. 2009. Morphology and classification of the Marchantiophyta. In: B. Goffinet \& A.J. Shaw (eds.). Bryophyte biology. Cambridge University Press, Cambridge, pp. 1-54.

Empain, A. 1973. La vegetation bryophytique aquatique et subaquatique de la Sambre Belge, son déterminisme écologique et ses relations avec la pollution des eaux. Lejeunia (novelle serie) 60: 1-58.

Flowers, S. 1973. Mosses: Utah and the West. Brigham Young University Press, Utah.

Glime, J. 1968. Ecological observations on some bryophytes in Appalachian Mountain streams. Castanea 33: 300-325.

Goffinet, B., Buck, W.R. \& Shaw, A.J. 2009. Morphology, anatomy and classification of the Bryophyta. In: B. Goffinet \& A.J. Shaw (eds.). Bryophyte biology. Cambridge University Press, Cambridge, pp. 55-138.

Gradstein, S.R. \& Costa, D.P. 2003. The Hepaticae and Anthocerotae of Brazil. Memoirs of The New York Botanical Garden 87: 1-318.

Gradstein, S.R. \& Ilkiu-Borges, A.L. 2009. Guide to the plants of Central French Guiana . Part 4. Liverworts and Hornworts. Memoirs of The New York Botanical Garden 76: 1-140.

Gradstein, S.R., Churchill, S.P. \& Salazar-Allen, N. 2001. Guide to the bryophytes of tropical America. Memoirs of The New York Botanical Garden 86: 1-577.

Hammer, Ø., Harper, D.A.T. \& Ryan, P.D. 2001. PAST: Paleontological Statistics Software Package for Education and Data Analysis, versão. 3.01. Paleontologia Electronica 4: 1-9.
IDESP - Instituto de Desenvolvimento Econômico, Social e Ambiental do Pará. 2015. SEPOF - Secretaria do Estado de Planejamento Orçamento e Finanças Estatística Municipal - São Domingos do Capim, 2015, Governo do Estado do Pará. Disponível em http:// www.idesp.pa.gov.br/pdf/estatisticaMunicipal/pdf/ sDomingosCapim.pdf (acesso em 10-VII-2015).

Ilkiu-Borges, A.L. 2000. Lejeuneaceae (Hepaticae) da Estação Científica Ferreira Penna, Caxiuanã, município de Melgaço, Pará. Dissertação de Mestrado, Faculdade de Ciências Agrárias do Pará, Belém.

Lisboa, R.C.L. 1991. Histórico da briologia na Amazônia brasileira. Boletim do Museu Paraense Emílio Goeldi, série Botânica 7: 69-77.

Lisboa, R.C.L. \& Ilkiu-Borges, A.L. 2001. Briófitas de São Luís do Tapajós, município de Itaituba, com novas adições para o Estado do Pará. Boletim do Museu Paraense Emílio Goeldi, série Botânica 17: 75-91.

Lisboa, R.C.L. \& Ilkiu-Borges, A.L. 2007. Uma Nova Avaliação da Brioflora da Reserva do Mocambo, Belém (PA). In: J.I. Gomes (org.). História Natural e Biologia da área de Pesquisa Ecológica do Guamá-Apeg. Museu Paraense Emílio Goeldi, EMBRAPA Belém, Belém.

Margurran, A.E. 1989. Diversidad Ecológica y su medición. Ediciones Vedrà, Barcelona.

McFarland, K.D. \& Wistendahl, W.A. 1976. Relationship of terrestrial mosses to forested flood plains in Southeastern Ohio. The Bryologist 79: 64-75.

Middleton, B.A. (ed.). 2002. Flood Pulsing in Wetlands: Restoring the Natural Hydrological Balance. Beth A. John Wiley \& Sons, Lafayette.

Ministério do Meio Ambiente. 2014. Amazônia. Disponível em http://www.mma.gov.br/biomas/ amaz\%C3\%B4nia (acesso em 30-VIII-2014).

Moraes, E.N.R. 2006. Diversidade, aspectos florísticos e ecológicos dos musgos (Bryophyta) da Estação Científica Ferreira Penna, Flona de Caxiuanã, Pará, Brasil. Dissertação de Mestrado. Universidade Federal Rural da Amazônia e Museu Paraense Emílio Goeldi, Belém.

Moraes, E.N.R. \& Lisboa, R.C.L. 2009. Diversidade, taxonomia e distribuição por estados brasileiros das famílias Bartramiaceae, Brachytheciaceae, Bryaceae, Calymperaceae, Fissidentaceae, Hypnaceae e Leucobryaceae (Bryophyta) da estação científica Ferreira Penna, Caxiuanã, Pará, Brasil. Acta Amazonica 39: 773-792.

Papp, B., Vanderpoorten, A. \& Gradstein, R. 2010. Sampling of bryophytes. In: J. Eyman, J. Degreef, C. Häuser, J.C. Monje, Y. Samyn \& D. Vanden Spiegel. ABC Taxa, n. 8, v. 1, pp. 340-354.

Proctor, M.C.F. 1982. Chapter 10: Physiological Ecology: Water relations, light and temperature responses, carbon balance. In: A.J.E. Smith (ed.). Bryophyte Ecology. 2 ed. Chapman and Hall, New York, pp. 333-382. 
Robbins, R.G. 1952. Bryophyte Ecology of a Dune Area in New Zealand. Vegetatio, Acta Geobotanica 4: 1-31.

Ruinen, J. 1953. Epiphytosis. A second view on epiphytism. Annales Bogorienses 1: 101-157.

Santos, R.C.P. 2006. Estudo comparativo da diversidade de musgos (Bryophyta) em diferentes ecossistemas da Microrregião do Salgado Paraense, Brasil. Dissertação de Mestrado. Universidade Federal Rural da Amazônia, Belém.

Sharp, A.J., Crum, H. \& Eckel, P. 1994. The moss Flora of Mexico. Memoirs of The New York Botanical Garden 69: 1-1113.
Silva, J.M.C., Rylands, A.B. \& Fonseca, G.A.B. 2005. $\mathrm{O}$ destino das áreas de endemismo da Amazônia. Megadiversidade 1: 124-131.

Veloso, H.P., Rangel Filho, A.L.R. \& Lima, J.C.A. 1991. Classificação da Vegetação Brasileira adaptada a um Sistema Universal. IBGE, Rio de Janeiro.

Wagner, S., Bader, M.Y. \& Zotz, G. 2014. Physiological Ecology of Tropical Bryophytes. In: D.T. Hanson \& S.K. Rice (eds.). Photosynthesis in Bryophytes and Early Land Plants. Springer, New York, pp. 269-290. 